\title{
Treino de marcha com suporte de peso corporal: diferentes protocolos melhoram o equilíbrio na Lesão Medular. ${ }^{1}$
}

\author{
Isadora Brites Rodrigues'; Camila Gabriela Haab³; Ellen Aguirre de Melo \\ Patricia Bastianello Palma ${ }^{5}$; Nadiesca Taísa Filippin ${ }^{6}$; Sheila Spohr Nedel'; \\ Juliana Saibt Martins ${ }^{8}$
}

\begin{abstract}
RESUMO
A lesão medular (LM) é causada por traumas diretos na medula espinhal ou em decorrência da compressão de vértebras. A LM causa alterações na sensibilidade e na função motora. O objetivo dessa pesquisa foi investigar na literatura a caracterização do treino de marcha com suporte de peso corporal empregado para melhorar o equilíbrio postural de indivíduos com LM. A coleta de dados foi realizada nas bases de dados eletrônicas National Library of Medicine (Pubmed) e Biblioteca Virtual de Saúde (BVS) e foram incluídos na amostra artigos publicados dos últimos 10 anos. Encontrou-se 8 artigos sendo que 6 foram incluídos na pesquisa por conta dos critérios de inclusão. Os resultados encontrados demonstram que o treino locomotor com suporte de peso corporal é seguro e confiável e mostra-se viável na reabilitação de pacientes que sofrem de uma lesão medular incompleta (ICSi). Trazendo benefícios referentes ao equilíbrio, caminhada, aumento da velocidade de marcha e aumento do comprimento do passo.
\end{abstract}

Palavras-chave: Lesão medular, equilíbrio, suporte de peso e treino locomotor.

Eixo Temático: Atenção Integral e Promoção à Saúde.

1 Trabalho de pesquisa.

2 Acadêmica do Curso de Fisioterapia-UFN isadora.rodrigues@ufn.edu.br

${ }^{3}$ Acadêmica do Curso de Fisioterapia - UFN camila.haab@ufn.edu.br

${ }^{4}$ Acadêmica do Curso de Fisioterapia -UFN ellen.melo@ufn.edu.br

${ }^{5}$ Acadêmica do Curso de Fisioterapia -UFN patricia.palma@ufn.edu.br

${ }^{6}$ Docente do curso de Fisioterapia-UFN nadifilippin@ufn.edu.br

${ }^{7}$ Docente do curso de Fisioterapia -UFN sheila.nedel@ufn.edu.br

${ }^{8}$ Orientadora. Docente do curso de Fisioterapia -UFN jsaibt@ufn.edu.br

\section{INTRODUÇÃO}

A lesão medular (LM) é causada, principalmente, por traumas diretos na medula espinhal ou em decorrência da compressão de vértebras deslocadas ou fraturadas (XIAODONG., 2021) devido a acidentes de trânsito e ao aumento da violência com armas brancas e de fogo (LIANZA., 2001). A incidência de LM no Brasil é de 40 casos 
novos ao ano por milhão de habitantes, ou seja, cerca de 6 a 8 mil novos casos a cada ano, tendo maior prevalência (80\%) no sexo masculino (GREVE, 1997).

A LM acarreta déficits sensoriais e motores, sexuais, urinários e intestinais (LIANZA, 2001), que variam e dependem do grau e nível da lesão, a qual pode ser classificada como completa ou incompleta. Em lesões completas, ocorre perda sensitiva e paralisia motora total abaixo do nível da lesão, devido à interrupção completa dos tratos nervosos, já nas incompletas estão preservados grupos musculares e áreas sensitivas (CEREZETTI, 2012).

Uma das principais expectativas dos pacientes com LM quanto ao tratamento é a recuperação da função motora, principalmente naqueles com lesão medular incompleta (ICSi) (ANDERSON, 2004). Para tanto, é necessário um programa de reabilitação com uma equipe multiprofissional, na qual estão inseridos os fisioterapeutas (HUBLI; DIETZ 2013).

Dentre as principais sequelas da LM está o prejuízo no equilíbrio, o que acarreta em distúrbios na mobilidade, postura e marcha (HUBLI; DIETZ 2013; HARKEMA et al., 2012; HORNBY et al., 2020; RUBIO et al., 2020). De fato, após uma LM, a capacidade de suportar o peso do corpo inteiro durante a marcha torna-se prejudicada, dificultando o treinamento locomotor (TL) dos pacientes acometidos. Dessa forma, o desenvolvimento de um sistema de colete ajustado ao corpo que suspende parte do peso corporal durante a marcha, o qual geralmente está acoplado a uma esteira motorizada, possibilitou esse treinamento. O referido dispositivo permite a prática de passos contínuos, sendo que muitas vezes os terapeutas necessitam fornecer assistência para facilitar padrões mais adequados de passos durante a prática (BARBEAU; WAINBERG; FINCH, 1987).

Alguns estudos comprovaram que o treino aeróbico de caminhada em esteira com intensidade moderada a alta acoplado a dispositivo de realidade virtual (VR) parece promover uma maior melhora no quadro locomotor dos pacientes (HUBLI, 2013; HARKEMA et al., 2012; HORNBY et al., 2020; RUBIO et al., 2020). O TL com suporte parcial de peso corporal enfatiza o exercício de alta intensidade para tarefas específicas, ativando e promovendo a plasticidade dos circuitos neuronais espinhais, 
podendo induzir à recuperação funcional da marcha em pacientes com LM (HUBLI, 2013; HARKEMA et al., 2012; HORNBY et al., 2020; RUBIO et al., 2020).

No entanto, os protocolos de TL diferem entre si em termos de duração, intensidade, velocidade da esteira, quantidade de peso suportado, entre outros aspectos. Assim, este estudo teve como objetivo buscar na literatura a caracterização do treino de marcha com suporte de peso corporal empregado para melhorar 0 equilíbrio postural de indivíduos com LM.

\section{MATERIAIS E MÉTODOS}

Este estudo caracteriza-se como exploratório, do tipo revisão integrativa da literatura. A busca dos estudos ocorreu no mês de setembro de 2021, através das bases de dados eletrônicas National Library of Medicine (Pubmed) e Biblioteca Virtual de Saúde (BVS). Como estratégia de busca, foram utilizados os seguintes descritores: "Locomotor Training" AND "Body Weight Support" AND "Spinal Cord Injury" AND "Postural Balance", e seus correspondentes na língua portuguesa e espanhola, os quais estão referenciados nos Descritores em Ciências da Saúde (DeCS) e Medical Subject Headings (MESH).

Os critérios de inclusão utilizados para selecionar os artigos foram: artigos publicados nos últimos 10 anos, disponíveis de forma completa e gratuita nos idiomas português, inglês ou espanhol e que abordassem o tema. Foram excluídos do estudo os artigos de revisão e pesquisas em modelo animal. A seleção dos estudos incluídos foi realizada em duas etapas distintas. Na primeira, realizou-se a busca nas bases de dados, a análise dos títulos e resumos. A segunda etapa consistiu na leitura da íntegra das publicações para confirmar se atendiam aos critérios de inclusão. Uma vez selecionados, os estudos foram categorizados por meio de um formulário planejado para coleta dos seguintes itens: título, autores/ano, intervenção e resultados.

\section{RESULTADOS}


Inicialmente a busca nas referidas bases de dados resultou em 8 artigos, todos da Pubmed. Após a análise dos títulos e resumos foram excluídos 2 artigos por fuga do tema. Na sequência à leitura da íntegra dos artigos, se manteve os 6 estudos conforme os critérios citados acima. A síntese dos 6 artigos se encontra na tabela 1.

Tabela 1: Características dos artigos selecionados nas bases de dados.

\begin{tabular}{|c|c|c|c|}
\hline $\begin{array}{c}\text { Autor/ano/ } \\
\text { amostra do } \\
\text { estudo }\end{array}$ & $\begin{array}{l}\text { Protocolo do } \\
\text { treinamento locomotor }\end{array}$ & $\begin{array}{l}\text { Instrumento de } \\
\text { avaliação do } \\
\text { desfecho }\end{array}$ & Resultados \\
\hline $\begin{array}{l}\text { COVARRUBIAS- } \\
\text { ESCUDERO, F } \\
\text { et al. (2019) } \\
17 \text { indivíduos, } 13 \\
\text { homens e quatro } \\
\text { mulheres }\end{array}$ & $\begin{array}{l}18 \text { sessões, três vezes } \\
\text { por semana, ao longo de } \\
\text { seis semanas. Cada } \\
\text { sessão consistia em três } \\
\text { séries de seis minutos de } \\
\text { TL em esteira, com dois } \\
\text { minutos de intervalo entre } \\
\text { cada série. A quantidade } \\
\text { adequada de suporte de } \\
\text { peso corporal foi } \\
\text { determinada de acordo } \\
\text { com o comprometimento } \\
\text { motor e a tolerância ao } \\
\text { exercício de cada } \\
\text { participante. A velocidade } \\
\text { inicial da esteira foi de } 0,5 \\
\text { km / h. }\end{array}$ & $\begin{array}{l}\text { Teste de equilíbrio } \\
\text { em pé (ISway) }\end{array}$ & $\begin{array}{l}\text { O equilíbrio em pé } \\
\text { melhorou após o } \\
\text { programa ( } p=0,016 \text { ), } \\
\text { mas nenhuma diferença } \\
\text { significativa foi } \\
\text { encontrada nos valores } \\
\text { raiz quadrada média } \\
\text { (RMS) para oscilação } \\
\text { postural ( } p=0,693 \text { ). } \\
\text { Nenhum dos pacientes } \\
\text { obteve melhora na } \\
\text { pontuação do WISCl II } \\
\text { antes e depois da } \\
\text { intervenção. }\end{array}$ \\
\hline
\end{tabular}




\begin{tabular}{|c|c|c|c|}
\hline $\begin{array}{l}\text { NAVARRETE } \\
\text { OPAZO, A et al. } \\
\text { (2016) } \\
35 \text { indivíduos }\end{array}$ & $\begin{array}{l}\text { Os participantes } \\
\text { receberam hipóxia } \\
\text { intermitente ou normoxia } \\
\text { contínua seguida de } 45 \\
\text { minutos de TL por } 5 \text { dias } \\
\text { consecutivos. Após foi } \\
\text { realizado } 3 \text { vezes por } \\
\text { semana, por } 3 \text { semanas } \\
\text { adicionais. O TL iniciou } \\
\text { com suspensão de } 30 \% \\
\text { do peso corporal e a } \\
\text { velocidade inicial da } \\
\text { esteira foi de } 0,6 \mathrm{~km} / \mathrm{h} \text {, } \\
\text { com facilitação manual } \\
\text { pelo terapeuta. }\end{array}$ & $\begin{array}{l}\text { Software de } \\
\text { mobilidade } \\
\text { (Ambulatory } \\
\text { Parkinson's } \\
\text { Disease } \\
\text { Monitoring's } \\
\text { Mobility Lab), } \\
\text { TUG, Teste de } \\
\text { equilíbrio em pé } \\
\text { (ISway), Timed Up } \\
\text { and Go (TUG) }\end{array}$ & $\begin{array}{l}\text { Não houve diferença no } \\
\text { equilíbrio em pé entre as } \\
\text { intervenções, porém os } \\
\text { indivíduos } \\
\text { receberam } \text { que } \\
\text { intermitente mais TL } \\
\text { mostraram melhora no } \\
\text { equilíbrio dinâmico } \\
\text { (cadência, giro e tempo } \\
\text { do giro para sentar). }\end{array}$ \\
\hline $\begin{array}{l}\text { HARKEMA, S et } \\
\text { al. } \\
(2011) \\
196 \text { indivíduos, } \\
\text { sendo } 148 \\
\text { homens e } 48 \\
\text { mulheres }\end{array}$ & $\begin{array}{l}\text { TL intensivo, incluindo } \\
\text { treinamento de passos } \\
\text { usando suporte de peso } \\
\text { corporal e facilitação } \\
\text { manual em uma esteira, } \\
\text { seguido por avaliação em } \\
\text { solo e integração com a } \\
\text { comunidade. }\end{array}$ & $\begin{array}{l}\text { Escala de } \\
\text { equilíbrio de Berg } \\
\text { (BBS), Teste de } \\
\text { caminhada de seis } \\
\text { minutos, Teste de } \\
\text { caminhada de } 10 \\
\text { metros (10MWT), }\end{array}$ & $\begin{array}{l}\text { As medidas de } \\
\text { resultados na inscrição } \\
\text { mostraram alta } \\
\text { variabilidade entre os } \\
\text { pacientes com American } \\
\text { Spinal Injury Association } \\
\text { Impairment } \\
\text { Scale (AIS) graus C e D. } \\
\text { Uma melhora } \\
\text { significativa desde a } \\
\text { inscrição até a avaliação } \\
\text { final foi observada nas } \\
\text { medidas de equilíbrio e } \\
\text { caminhada para } \\
\text { pacientes com AIS graus } \\
\text { C e D. A magnitude da } \\
\text { melhora diferiu } \\
\text { significativamente entre } \\
\text { os grupos AIS para todas } \\
\text { as medidas. }\end{array}$ \\
\hline
\end{tabular}




\begin{tabular}{|c|c|c|c|}
\hline $\begin{array}{l}\text { FOSTER, H et al. } \\
(2016) \\
\text { Uma mulher com } \\
28 \text { anos }\end{array}$ & $\begin{array}{l}18 \text { sessões de } 60 \text { minutos } \\
\text { de treinamento de } \\
\text { caminhada para trás em } \\
\text { uma esteira com suporte } \\
\text { de peso corporal parcial e } \\
\text { solo, realizado três vezes } \\
\text { por semana durante } 6 \\
\text { semanas. }\end{array}$ & $\begin{array}{l}\text { Escala de } \\
\text { equilíbrio de Berg } \\
\text { (BBS), Teste de } \\
\text { organização } \\
\text { sensorial (SOT), } \\
\text { Tester de } \\
\text { caminhada de } 10 \\
\text { metros (10MWT), } \\
\text { teste de } \\
\text { caminhada para } \\
\text { trás de } 3 \text { metros, } \\
\text { Timed Up and Go } \\
\text { (TUG) e Escala de } \\
\text { confiança de } \\
\text { equilíbrio (ABC). }\end{array}$ & $\begin{array}{l}\text { A melhora do equilíbrio } \\
\text { foi evidente com base } \\
\text { nas pontuações BBS ( } 20 \\
\text { a 37/56) e SOT ( } 27 \text { a } \\
40 / 100 \text { ). A mobilidade } \\
\text { ereta melhorou (TUG de } \\
57 \text { para } 32,7 \text { s), } \\
\text { aumentou a velocidade } \\
\text { de 10MWT (0,23 a 0,31 m } \\
\text { / s) e a velocidade de } \\
\text { marcha para trás ( } 0,07 \text { a } \\
0,12 \mathrm{~m} / \mathrm{s} \text { ). Além disso, a } \\
\text { pontuação na ABC } \\
\text { aumentou de } 36,9 \% \text { para } \\
49,6 \% \text {. }\end{array}$ \\
\hline 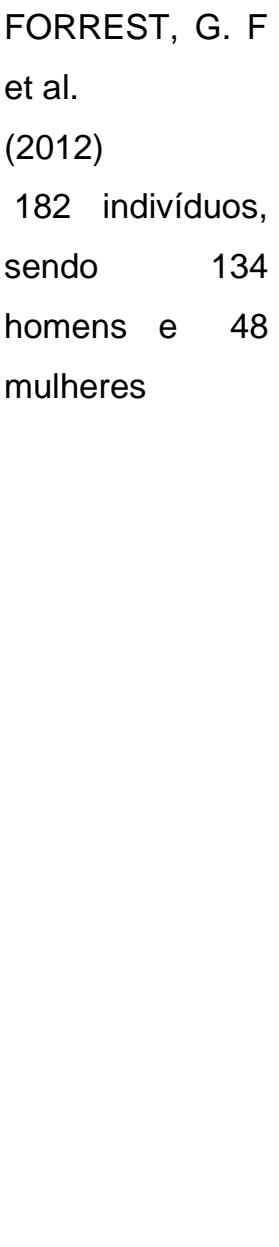 & $\begin{array}{l}\text { TL intensivo em uma } \\
\text { esteira utilizando suporte } \\
\text { parcial de peso corporal e } \\
\text { facilitação manual pelo } \\
\text { terapeuta. O TL era } \\
\text { realizado por } 60 \text { minutos, } \\
\text { de } 3 \text { a } 5 \text { vezes por } \\
\text { semana por nove } \\
\text { semanas. }\end{array}$ & $\begin{array}{l}\text { Teste de } \\
\text { caminhada de seis } \\
\text { minutos, Teste de } \\
\text { caminhada de } 10 \\
\text { metros (10MWT), } \\
\text { Escala de } \\
\text { equilíbrio de Berg } \\
\text { (BBS), Alcance } \\
\text { Funcional } \\
\text { Modificado }\end{array}$ & $\begin{array}{l}\text { As medidas de } \\
\text { caminhada e equilíbrio } \\
\text { em pé para todos os } \\
\text { participantes foram } \\
\text { fortemente } \\
\text { correlacionadas, as } \\
\text { medidas de equilíbrio em } \\
\text { pé e sentado não foram } \\
\text { altamente } \\
\text { correlacionadas e as } \\
\text { medidas de caminhada } \\
\text { foram fracamente } \\
\text { relacionado ao equilíbrio } \\
\text { sentado. A força das } \\
\text { relações entre as } \\
\text { medidas de resultado } \\
\text { variou com o estado } \\
\text { funcional. Os testes de } \\
\text { caminhada, quando } \\
\text { realizados com diferentes } \\
\text { dispositivos de }\end{array}$ \\
\hline
\end{tabular}




\begin{tabular}{|c|c|c|c|}
\hline & & & $\begin{array}{l}\text { assistência, foram } \\
\text { fortemente } \\
\text { correlacionados, mas } \\
\text { tiveram uma variabilidade } \\
\text { substancial no } \\
\text { desempenho. }\end{array}$ \\
\hline $\begin{array}{l}\text { MORIELLO, G. } \\
\text { et al (2018) } \\
\text { Um homem com } \\
38 \text { anos }\end{array}$ & $\begin{array}{l}24 \text { sessões, realizadas } \\
\text { três vezes por semana } \\
\text { por } 60 \text { minutos, sendo } 30 \\
\text { minutos de } \\
\text { retreinamento de passo, } \\
15 \text { minutos de } \\
\text { adaptabilidade de etapa e } \\
15 \text { minutos de } \\
\text { adaptabilidade do } \\
\text { suporte. Também foi } \\
\text { solicitado que o indivíduo } \\
\text { realizasse } \\
\text { treinamento um } \\
\text { transferência de peso, } \\
\text { controle postural } \mathrm{e} \\
\text { marcha durante } 30 \\
\text { minutos em casa. }\end{array}$ & $\begin{array}{l}\text { Escala de } \\
\text { equilíbrio de Berg } \\
\text { (BBS), Teste de } \\
\text { caminhada de seis } \\
\text { minutos, } \\
\text { parâmetros } \\
\text { espaço-temporais } \\
\text { da marcha }\end{array}$ & $\begin{array}{l}\text { A pontuação BBS } \\
\text { melhorou de } 37 / 56 \text { para } \\
\text { 43/56. O comprimento do } \\
\text { passo esquerdo } \\
\text { melhorou; embora a } \\
\text { velocidade da marcha, } \\
\text { cadência, comprimento } \\
\text { da passada e } \\
\text { comprimento do passo } \\
\text { direito não o fizeram. } \\
\text { Mudanças observáveis } \\
\text { foram notadas na } \\
\text { qualidade da marcha. A } \\
\text { distância de caminhada } \\
\text { de seis minutos } \\
\text { aumentou em } 47,2 \text { m. }\end{array}$ \\
\hline
\end{tabular}

\section{DISCUSSÃO}

A reabilitação de pessoas com LM é multidisciplinar e envolve vários profissionais de saúde, ela precisa ser iniciada precocemente. A fisioterapia é a abordagem da reabilitação mais importante para a recuperação da locomoção, da força muscular, da sensibilidade e para o aumento do equilíbrio, entre outras (DIETZ., 1995). Além disso, a reabilitação locomotora depende do sistema nervoso, que se adapta às atividades específicas, e a reaprendizagem da deambulação como tarefa 
específica traz recuperação pois o treinamento de marcha com velocidade proporciona informação sensorial para a medula espinhal (EDGERTON; et al. 1991).

Covarrubias et al. (2019), encontraram diferenças significativas no equilíbrio em pé dos pacientes com LM após o programa. No estudo de Foster et al (2016) também foi identificada uma melhora do equilíbrio com base nas pontuações na Escala de Equilíbrio de Berg e SOT. Já no estudo de Navarrete e colaboradores (2016), não houve diferença significativa no equilíbrio em pé entre as intervenções.

No estudo de Harkema e colaboradores (2011), os resultados mostraram diferenças entre os pacientes com AIS graus C e D, já para as medidas de equilíbrio e caminhada para os mesmos, ocorreu uma melhora significativa desde a avaliação inicial até a final, a relevância de melhora entre os dois grupos diferiu em todas as medidas.

Apenas no estudo de Moriello, et al (2018), foi notado que o comprimento do passo esquerdo foi melhorado, mas o comprimento da passada e comprimento do passo direito não melhoraram. Porém, mudanças foram notadas na qualidade da marcha e na distância de caminhada de seis minutos. No estudo de Forrest et al (2012), as medidas de caminhada foram fracamente relacionadas ao equilíbrio sentado, variando com o estado funcional. O teste de caminhada de seis minutos e o teste de caminhada de 10 metros (10MWT), quando realizados com diferentes dispositivos de assistência, foram fortemente correlacionados, mas tiveram uma variabilidade substancial no desempenho. O aumento do comprimento do passo pode ser explicado pelo aumento da extensão do quadril e da flexão plantar, melhorando também o tempo de apoio e tempo de balanço (GAGE, 2004; MILLER, 2007).

Estudos clínicos randomizados foram realizados por Postans (2004); Field-Fote (2005); Dobkin (2006), a fim de comparar o treino de marcha com suporte de peso com outras modalidades terapêuticas para a melhora da locomoção e os resultados foram semelhantes em ambos os grupos, melhorando a independência, capacidade de deambulação e aumento da velocidade da marcha em pacientes com LM.

Os protocolos de cada estudo mostraram variações quanto à frequência e número de sessões. O estudos de Foster et al. (2016) e de Covarrubias-Escudero et al. (2019) incluíram 18 sessões, realizadas três vezes por semana, na pesquisa de 
Forrest et al. (2012) foram 20 sessões. Já no estudo do Moriello (2018) aplicou-se 24 sessões, porém no estudo do Harkema et al (2011) consta 47 sessões.

Os estudos utilizaram diferentes instrumentos de medida para avaliar o efeito do TL sobre o equilíbrio de indivíduos com LM, porém a escala de equilíbrio de Berg (BBS) e o teste de caminhada de seis minutos (TC6) foram os mais empregados. 0 TC6 é um teste de fácil realização e consiste em avaliar as diversas funções corporais, entre elas o desempenho do paciente durante um exercício, possibilitando a análise da aptidão física e a capacidade funcional (PEREIRA, L.; VIEIRA, K; TEIXEIRA, P; 2018). O BSS avalia o equilíbrio dinâmico e estático dos indivíduos e o risco de quedas considerando a influência ambiental na função, ele consiste em 14 testes sendo estes direcionados para a habilidade do indivíduo de sentar, ficar de pé, alcançar, girar em volta de si mesmo, olhar por cima de seus ombros, ficar em apoio unipodal e transpor degraus (MIYAMOTO et al., 2004).

\section{CONCLUSÃO}

Conforme a análise dos artigos, o treino de marcha com suporte de peso corporal tem sido utilizado para melhorar o equilíbrio postural de indivíduos com LM por meio de protocolos diversos, os quais diferem entre si principalmente quanto ao número de sessões realizadas.

\section{REFERÊNCIAS}

ANDERSON KD. Objetivando a recuperação: prioridades da população com lesões na medula espinhal . J Neurotrauma, v.21, n. 10, p. 1371-83, 2004.

BARBEAU, H; WAINBERG, M.; FINCH, L. Description and application of a system for locomotor rehabilitation. Medical \& Biological engineering \& Computing, v. 25, p. 341-344, 1987.

CEREZETTI, $C$ et al. Lesão Medular Traumática e estratégias de enfrentamento: revisão crítica. O Mundo da Saúde, v. 36, n. 2, p. 318-326, 2012. 
COVARRUBIAS-ESCUDERO, F et al. Effects of body weight-support treadmill training on postural sway and gait independence in patients with chronic spinal cord injury. $\mathbf{J}$ Spinal Cord Med, v. 42, n. 1, p. 57-64, 2017.

DOBKIN, B.; APPLE, D. et al. Weight-supported treadmill vs overground training for walking after acute incomplete SCI. Neurology, v.66, n.4, p.484-492. 2006.

EDGERTON, V.R.; ROY, R.R. et al. A physiological basis for the development of rehabilitative strategies for spinally injured patients. J Am Paraplegia Soc, v.14, n.4. p.150-157, 1991.

FIELD-FOTE, E. C. Combined use of body weight support, functional electric stimulation, and treadmill training to improve walking ability in individuals with chronic incomplete spinal cord injury. Arch Phys Med Rehabil, v.82, n.6, p.818 824. 2001.

FOSTER, $\mathrm{H}$ et al. The effects of backward walking training on balance and mobility in an individual with chronic incomplete spinal cord injury: A case report. Physiother Theory Pract, v. 32, n. 7, p. 536-45, 2016.

FORREST, G et al. Ambulation and balance outcomes measure different aspects of recovery in individuals with chronic, incomplete spinal cord injury. Arch Phys Med Rehabil, v. 93, n. 9, p. 1553-64, 2011.

GAGE, J. The treatment of gait problems in cerebral palsy. Clinics in deselopmental medicine, n. 164, p. 448, 2004.

GREVE, J. Traumatismos raquimedulares nos acidentes de trânsito e uso de equipamentos de segurança. Diag. \& Tratam, v. 2, n. 3, p. 10-13, 1997.

HARKEMA SJ, et al. Locomotor training: as a treatment of spinal cord injury and in the progression of neurologic rehabilitation. Arquivos de Medicina Física e Reabilitação, v. 93, p. 1588-1597, 2012.

HARKEMA, S et al. Balance and ambulation improvements in individuals with chronic incomplete spinal cord injury using locomotor training-based rehabilitation. Arch Phys Med Rehabil, v. 93, n. 9, p. 1508-17, 2011.

HORNBY, TG et al. Clinical Practice Guideline to Improve Locomotor Function Following Chronic Stroke, Incomplete Spinal Cord Injury, and Brain Injury. J Neurol Phys Ther, v. 44, n. 1, p. 49-100, 2020.

HUBLI, M.; DIETZ, V.. As bases fisiológicas do treinamento locomotor de neurorreabilitação após lesão medular . J Neuroeng Rehabil, v. 10, n. 5, p. 1-8, 2013.

PEREIRA, L.; VIEIRA, K; TEIXEIRA, P. Teste de caminhada de 6 minutos e sua aplicabilidades: uma revisão sistemática da literatura. Revista Valore, v.3, n .2, p. 646-662, 2018. 
LIANZA, S.; CASALIS, ME, GREVE JMD, et al. A lesão medular. Medicina de Reabilitação. Rio de Janeiro: Guanabara Koogan, p. 299-322, 2001.

MILLE, F. Physical therapy of cerebral palsy. Softcover, p. 416, 2007.

MIYAMOTO, S.T et al. Brazilian version of the Berg balance scale. Brazilian Journal of Medical and Biological Research, v.37, n.19, p.1411-1414, 2004.

MORIELLO, G et al. Outcomes following a locomotor training protocol on balance, gait, exercise capacity, and community integration in an individual with a traumatic brain injury: a case report. Physiother Theory Pract, v. 35, n. 12, p. 1343-1354, 2019.

NAVARRETE-OPAZO, A et al. Intermittent Hypoxia and Locomotor Training Enhances Dynamic but Not Standing Balance in Patients With Incomplete Spinal Cord Injury. Arch Phys Med Rehabil, v. 98, n. 3, p. 415-424, 2016.

POSTANS, N.J.; HASLER, J.P. et al. Functional electric stimulation to augment partial weight-bearing supported treadmill training for patients with acute incomplete spinal cord injury: A pilot study. Arch Phys Med Rehabil, v.85. n.4. p.604-10. 2004.

RUBIO, A et al. Is Virtual Reality Effective for Balance Recovery in Patients with Spinal Cord Injury? A Systematic Review and Meta-Analysis. J Clin Med, v. 9, n. 9, p. 2861, 2020

XIAODONG,G.; YAPING, F.; TIANSHENG, S, et al. Clinical guidelines for neurorestorative therapies in spinal cord injury. Journal of Neurorestoratology, $\vee 9$, n 1, p 31-49, 2021. 